\title{
Tratamento da pseudartrose infectada da tíbia pelo método de Ilizarov: técnica do encurtamento agudo com subseqüente alongamento*
}

\author{
Treatment of infected pseudoarthrosis of the tibia \\ by Ilizarov's method: technique using acute \\ compression and subsequent distraction
}

\author{
Jorge LUIZ Borges ${ }^{\dagger}$, OSMAR LOPES JÚNIOR ${ }^{1}$, JUNG Ho KIM², CARLO MILANI ${ }^{3}$
}

\section{RESUMO}

Objetivo: Comprovar a eficácia do tratamento da pseudartrose infectada da tíbia pelo método de Ilizarov, utilizando a técnica do encurtamento agudo do foco da pseudartrose com posterior alongamento ósseo. Métodos: Foram estudados 20 pacientes adultos portadores de pseudartrose unilateral infectada da tíbia, tratados no período entre novembro de 1993 e setembro de 2000. No pós-operatório, os pacientes foram avaliados quanto a dor, necessidade de órtese, avaliação da mobilidade articular, retorno às atividades normais do dia-adia e/ou do trabalho, consolidação da pseudartrose, cura da infecção, existência de deformidades ósseas, discrepância no comprimento dos membros e consolidação óssea na área do regenerado ósseo. Resultados: Os resultados funcionais foram: excelentes em 10 pacientes (50\%), bons em oito (40\%), regulares em um (5\%) e ruins em um paciente $(5 \%)$. 0 tempo de

* Tese apresentada à Universidade Federal de São Paulo - Escola Paulista de Medicina para obtenção do título de Doutor em Medicina. O título foi obtido post-mortem.

$\dagger$ Doutor pela Escola Paulista de Medicina - Universidade Federal de São Paulo - UNIFESP - São Paulo (SP), Brasil. In memoriam.

1. Instrutor da Residência Médica do Instituto de Ortopedia e Traumatologia - Passo Fundo (RS), Brasil.

2. Instrutor da Residência Médica e Chefe do Serviço de Ortopedia Pediátrica do IOT - Passo Fundo (RS), Brasil.

3. Livre-Docente; Professor Associado da Universidade Federal de São Paulo - UNIFESP - São Paulo (SP), Brasil; Professor Titular da Faculdade de Medicina do ABC - FMABC - Santo André (SP), Brasil.

Endereço para correspondência: Instituto de Ortopedia e Traumatologia, Rua Uruguai, 2.050 - 99010-112 - Passo Fundo (RS), Brasil. Tel.: (54) 3045-2000. E-mail: ovlopesjr@yahoo.com.br ${ }^{(2)}$.

Recebido em 15/8/05. Aprovado para publicação em 6/9/07.

Copyright RBO2007 consolidação da pseudartrose, do segmento ósseo alongado e o tempo de uso do fixador foram proporcionais à extensão da perda óssea. Conclusão: $O$ método de Ilizarov, associado ao encurtamento agudo com subsequiente alongamento ósseo, mostrou-se eficaz para o tratamento da pseudartrose infectada da tíbia.

Descritores - Pseudartrose/cirurgia; Técnica de Ilizarov; Fixadores externos/utilização; Osteomielite; Tibia/cirurgia

\section{ABSTRACT}

Objective: To verify the effectiveness of treating the infected pseudoarthrosis of the tibia by Ilizarov's method using the technique of acute compression of the focus of pseudoarthrosis with later bone distraction. Methods: 20 adult patients with unilateral infected pseudoarthrosis of the tibia treated from November 1993 to September 2000 were studied. In the postoperative period, the patients were evaluated for pain, orthesis requirement, joint mobility, return to daily life activities and/or to work, union of the pseudoarthrosis, cure of the infection, presence of bone deformities, discrepancy in limb length, and bone union in the bone regenerate area. Results: Functional results were: excellent in 10 patients (50\%), good in eight (40\%), regular in one (5\%), and poor in one patient (5\%). The time to achieve union of the pseudoarthrosis of the distracted bone segment and the time of use of the fixator were proportional to the extension of bone loss. Conclusion: Ilizarov's method associated to acute compression followed by bone distraction showed to be effective to treat infected pseudoarthrosis of the tibia.

Keywords - Pseudarthrosis/surgery; Ilizarov technique; External fixators/utilization; Osteomyelitis; Tibia/surgery 


\section{INTRODUÇÃO}

Segundo Crenshaw, a pseudartrose infectada da tíbia deve ser definida como uma fratura que não obteve consolidação óssea por mais de seis meses e que apresenta mobilidade no local da fratura ao exame clínico, com a presença de fístula secretante, pressupondo a presença de osso necrosado, seqüestro ósseo, osteomielite extensiva, lesão de tecidos moles, deformidade e discrepância de comprimento( ${ }^{(1)}$.

Ainda não existe consenso entre os ortopedistas quanto ao melhor tratamento, porém a maioria dos especialistas acredita que a ressecção radical dos tecidos desvitalizados, a estabilização mecânica adequada e o uso de enxerto ósseo esponjoso sejam indispensáveis para a cura da pseudartrose.

Há a necessidade de um tratamento que promova consolidação óssea, trate a infecção e resolva, concomitantemente, os problemas de discrepância de comprimento e deformidades angulares.

Ilizarov desenvolveu, na década de 50, um método para o tratamento dos pacientes que apresentavam pseudartrose infectada dos ossos da perna com ou sem defeito ósseo( ${ }^{(2)}$. O método de Ilizarov permite solucionar, simultaneamente, o encurtamento do membro, a deformidade angular, a perda óssea, a infecção, a perda de tecidos moles, além de outros problemas como as contraturas articulares ${ }^{(3-7)}$.

Para a maioria dos autores que utilizam o método de Ilizarov, a pseudartrose infectada da tíbia com perda óssea maior que $3 \mathrm{~cm}$ e nos casos secundários ao trauma inicial, às cirurgias prévias ou à ressecção no momento da reconstrução cirúrgica deve ser tratada com o transporte ósseo. Entretanto, muitos desses autores têm relatado alta taxa de complicações e necessidade de procedimentos cirúrgicos após a técnica do transporte ósseo ${ }^{(5,8-9)}$. Para evitar essas complicações, os tecidos desvitalizados (ósseo e partes moles) do local da pseudartrose devem ser ressecados, seguindo-se o encurtamento agudo (compressão imediata) no foco e o subseqüente alongamento gradual do membro $^{(4,6)}$.

\section{MÉTODOS}

O estudo incluiu 20 pacientes portadores de pseudartrose unilateral infectada da tíbia que foram tratados pelo método de Ilizarov, utilizando a técnica de encurtamento agudo do foco da pseudartrose com posterior alongamento, durante o período de novembro de 1993 a setembro de 2000. Os pacientes foram operados no Hospital São Vicente de Paulo pela Equipe de Fixadores Externos do Instituto de Ortopedia e Traumatologia de Passo Fundo, RS. Dos pacientes, 16 eram ho- mens e quatro eram mulheres, com média de idade de 32 anos (17-74). Quanto ao lado acometido, nove (45\%) foram do lado direito e $11(55 \%)$ do lado esquerdo. A média de tempo de evolução da pseudartrose foi de 13 meses (6-32 meses).

Quanto ao tipo do traço de fratura, 15 pacientes (75\%) tinham traço cominutivo e cinco $(25 \%)$ possuíam traço transverso ou oblíquo curto. Dos pacientes, oito (40\%) foram vítimas de acidente automobilístico; oito (40\%), de acidente por motociclismo; três (15\%), de quedas de altura; e um (5\%), de trauma em jogo de futebol. Quanto ao local da pseudartrose, em três pacientes (15\%), o foco encontrava-se no terço proximal; em 15 (75\%), no terço médio da tíbia; e, em dois (10\%), no terço distal. As pseudartroses foram distribuídas segundo a classificação de Ilizarov, modificada por Catagni ${ }^{(8)}$, como tipo A1 em três pacientes, tipo B1 em cinco, tipo B2 em sete e tipo $\mathrm{B} 3$ em cinco. No que se refere às cirurgias prévias realizadas, dois pacientes $(10 \%)$ haviam sofrido mais de cinco procedimentos cirúrgicos; dois pacientes, quatro (10\%); oito pacientes, três (40\%); sete, dois (35\%), e um paciente, uma cirurgia $(5 \%)$. Os dados demográficos individualizados estão mostrados na tabela 1 .

TABELA 1

Dados demográficos

\begin{tabular}{clccccccc}
\hline Caso & Nome & $\begin{array}{c}\text { Idade } \\
\text { (anos) }\end{array}$ & Sexo & Lado & Traço & CP & LP & CL \\
\hline & & & & & & & \\
1 & MZ & 45 & M & D & C & 2 & M & B3 \\
2 & CAS & 27 & F & D & C & 3 & M & B1 \\
3 & GD & 32 & M & D & OC & 4 & M & A1 \\
4 & ALS & 36 & M & D & C & 3 & P & B2 \\
5 & JCV & 38 & M & D & C & 2 & M & B2 \\
6 & NO & 26 & M & E & OC & 3 & M & A1 \\
7 & PAC & 30 & M & D & C & 2 & M & B1 \\
8 & PP & 40 & M & E & C & 4 & M & B1 \\
9 & FN & 43 & M & E & C & 8 & P & B3 \\
10 & DM & 17 & F & E & OC & 2 & M & A1 \\
11 & RD & 37 & F & D & OC & 3 & M & B1 \\
12 & IK & 43 & F & E & C & 3 & M & B1 \\
13 & CM & 18 & M & E & C & 1 & P & B2 \\
14 & JS & 27 & M & D & C & 2 & M & B3 \\
15 & IC & 74 & M & E & C & 3 & D & B3 \\
16 & ELA & 27 & M & E & C & 2 & M & B3 \\
17 & CH & 18 & M & D & OC & 5 & M & B2 \\
18 & JJBS & 35 & M & E & C & 3 & M & B2 \\
19 & EC & 22 & M & E & C & 2 & D & B2 \\
20 & EG & 21 & M & E & C & 3 & M & B2 \\
& & & & & & & & \\
\hline
\end{tabular}

$\mathrm{CP}$ - cirurgias prévias; $\mathrm{LP}$ - local da pseudartrose; $\mathrm{CL}$ - classificação da pseudartrose; $\mathrm{M}$ - masculino; $\mathrm{F}$ - feminino; $\mathrm{C}$ - cominutiva; $\mathrm{OC}$ - transversa/oblíqua curta; $\mathrm{P}$ - proximal; M - médio; D - distal.

Fonte: Prontuário do IOT/RS e Hospital São Vicente de Paulo/RS. 
Com relação à infecção no foco da pseudartrose, utilizamos a classificação proposta pelo grupo $\mathrm{AO}^{(8)}$ e observamos que 12 pacientes $(60 \%)$ apresentavam infecção ativa com fístula; cinco (25\%), infecção quiescente; e três (15\%), infecção ativa, porém sem fístula.

$\mathrm{Na}$ avaliação das condições locais dos tecidos moles ao redor da pseudartrose, foi constatado em seis pacientes (30\%) um revestimento cutâneo adequado sobre a área da pseudartrose. Em nove casos (45\%), observou-se cobertura com tecido cicatricial de má qualidade com exposição óssea presente e, em cinco pacientes (25\%), foi encontrada exposição óssea, sem cobertura cutânea em toda a extensão da área lesada.

Os desvios angulares foram registrados em 12 pacientes $(60 \%)$, sendo que sete (35\%) apresentaram desvio em valgo, dois (10\%), desvio em varo, um (5\%), desvio em antecurvato e um (5\%), desvio em retrocurvato.

A discrepância do comprimento dos membros estava presente em 12 pacientes, variando de 1 a $3 \mathrm{~cm}$, com média de $1,3 \mathrm{~cm}$.

A perda óssea decorrente do trauma inicial ou de cirurgias prévias foi de $1 \mathrm{~cm}$ em sete (35\%) pacientes, $2 \mathrm{~cm}$ em nove (45\%) pacientes e de $1 \mathrm{~cm}$ em um (5\%) paciente. A média da perda óssea foi de $1,4 \mathrm{~cm}$. Apenas três pacientes não apresentavam perda óssea.

Na avaliação da limitação da mobilidade articular, dois pacientes (10\%) tinham limitação do arco de movimento do joelho, nove (45\%) do tornozelo.

As radiografias do membro comprometido foram obtidas na incidência ântero-posterior e lateral da perna (figura 1).

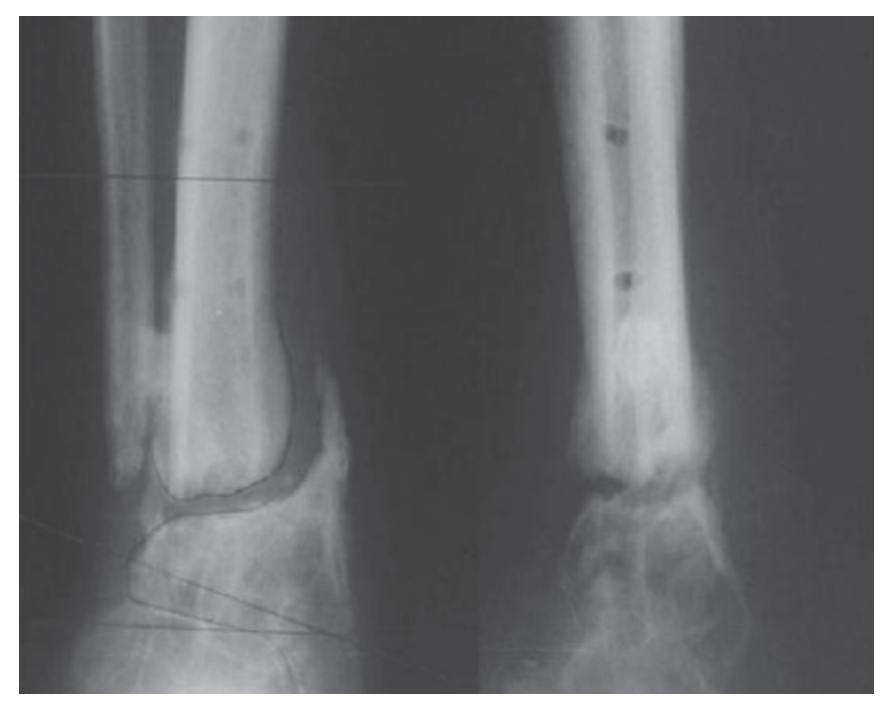

Figura 1 - Imagem radiológica pré-operatória de pseudartrose infectada da tíbia distal

\section{Técnica cirúrgica}

Inicialmente, os tecidos moles e osso necrosado e infectado foram cuidadosamente desbridados e enviados para o laboratório para a realização da cultura e antibiograma. Todos os pacientes receberam uma dose de $2 \mathrm{~g}$ de cefalotina após a colheita do material e mantida $0,5 \mathrm{~g}$ a cada seis horas. $\mathrm{O}$ antibiótico foi substituído, ou não, conforme o resultado do antibiograma, sendo mantido por mais 10 dias.

O segundo passo - osteotomia da fíbula - foi realizada em relação à localização do foco da pseudartrose: quando este se encontrava no terço proximal, a fíbula foi osteotomizada em dois níveis e o segmento ósseo foi descolado e removido; quando se situava no terço médio, a fíbula foi ressecada no mesmo nível, por meio de uma incisão longitudinal póstero-lateral e, quando no terço distal, esse procedimento foi realizado através da ferida operatória após a retirada da tíbia.

A estabilização provisória da tíbia foi conseguida pela passagem de um fio-guia intramedular (Kirschner $3 \mathrm{~mm}$ de diâmetro), que penetrou pelo maléolo medial em direção ao canal medular.

O aparelho de Ilizarov foi então instalado respeitando o eixo anatômico da perna. Posteriormente, o membro foi encurtado agudamente, até o contato completo do coto proximal da tíbia com o distal, sem ressecção adicional da pele excedente. A extensão do encurtamento agudo foi limitada pela vascularização do pé, avaliada por exame clínico ou com auxílio do doppler transoperatório. Na deficiência de vascularização, suspendia-se o encurtamento agudo e partiríamos para o encurtamento gradual de $1 \mathrm{~mm}$ por dia ao mesmo tempo do alongamento proximal.

A corticotomia ou osteotomia da tíbia para alongamento foi realizada nas regiões metafisárias proximal ou distal, a uma distância, aproximadamente, de $3 \mathrm{~cm}$ dos respectivos anéis, por meio de duas técnicas distintas. Em 10 pacientes (50\%), utilizamos perfurações com broca 3,5mm e osteótomo. Em outros 10 pacientes (50\%), realizamos osteotomia usando a serra de Gigli disposta subperiostalmente.

Por fim, idealizamos a montagem do aparelho de acordo com a localização da pseudartrose.

Os pacientes permaneceram hospitalizados por três dias. $\mathrm{O}$ alongamento foi iniciado em média entre o quarto e o sétimo dia pós-operatório, com progressão de 1/4 de milímetro a cada seis horas, totalizando $1 \mathrm{~mm}$ por dia, segundo o método preconizado por Ilizarov ${ }^{(2)}$. Os pacientes foram incentivados a praticar exercícios ativos e passivos do membro em sua residência. Retornaram para avaliação clínica e radiológica no 
final da primeira semana e, após, mensalmente (figura 2). Caso não houvesse formação de imagem de regeneração óssea nas radiografias de controle, o paciente era orientado a diminuir o ritmo e a taxa de alongamento para $1 / 4$ de volta a cada 12 horas.
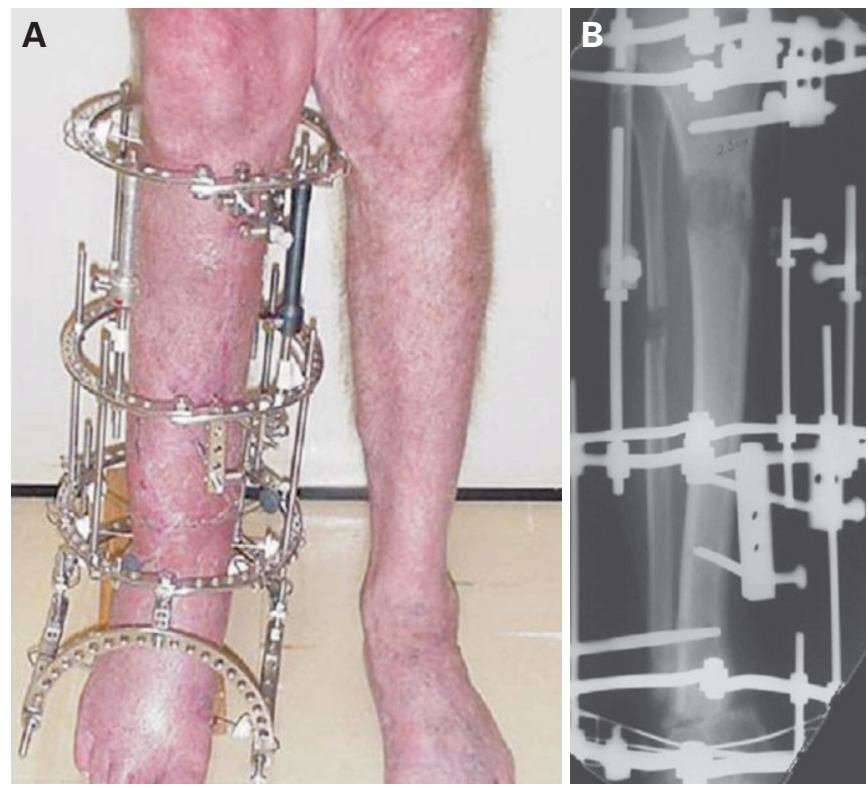

Figura 2 - Aspecto clínico (A) e radiológico (B) com seis meses de seguimento do paciente apresentado na figura 1

Para a retirada do aparelho de Ilizarov, foi realizado o teste clínico-radiológico de maneira rotineira. Radiograficamente, seguiram-se os critérios de consolidação óssea proposto por Fischgrund et $_{\text {al }}{ }^{(10)}$ e, quando a pseudartrose se mostrava consolidada, todas as hastes de conexão do foco eram soltas e testavam-se, clinicamente, a mobilidade e a presença de dor no foco da pseudartrose. Além disso, permitimos ao paciente deambular com carga por algumas horas, dias ou semanas com o aparelho solto. Se, após esse período, não fosse constatada mudança radiográfica no acompanhamento da consolidação, era, então, programada a retirada do fixador.

\section{Metodologia para avaliação dos resultados}

Os resultados clínicos e radiográficos foram analisados segundo os critérios propostos por Paley et $a l^{(5)}$.

\section{1) Critérios para a avaliação funcional}

Os resultados funcionais foram baseados em cinco critérios: dor; necessidade de órtese para ajudar na deambulação; perda da mobilidade articular do joelho, tornozelo e pé com- parada com a mobilidade existente pré-operatória; retorno às atividades normais do dia-a-dia e/ou do trabalho. Segundo esses critérios, os pacientes foram agrupados em padrões esperados: excelente: quando o paciente não apresentou dor (não precisando de medicação analgésica); não necessitou de órtese para deambulação; não teve deformidade; não apresentou contratura do joelho maior que $5^{\circ}$; não perdeu mais de $20^{\circ}$ na mobilidade das articulações comparada com a mobilidade préoperatória; foi capaz de realizar as atividades do dia-a-dia sem dificuldades; bom: quando o paciente não sentiu dor ou esta era esporádica; foi capaz de realizar as atividades do dia-a-dia com dificuldade mínima e apresentou falha em um dos outros critérios; regular: quando o paciente não teve dor ou esta era moderada; foi capaz de realizar as atividades do dia-a-dia com dificuldade mínima e apresentou falha em dois dos outros critérios; mau: o paciente sentiu dor constante significante, necessitando de narcóticos diários; foi incapaz de realizar as atividades do dia-a-dia e apresentou falha nos outros três critérios.

\section{2) Critérios para a avaliação radiográfica}

Os resultados ósseos foram baseados em cinco critérios: existência de consolidação da pseudartrose; cura da infecção e resolução das deformidades ósseas; discrepância no comprimento do membro; remodelação da área do regenerado ósseo e do encontro dos fragmentos ósseos.

O resultado ósseo geral foi considerado: excelente: consolidação óssea, nenhuma evidência de infecção, deformidade angular menor ou igual a $7^{\circ}$, em qualquer plano, discrepância dos membros inferior a $2,5 \mathrm{~cm}$ e consolidação óssea suficiente para não necessitar do uso de órtese; bom: consolidação óssea sem infecção e falha em encontrar um dos outros critérios; regular: consolidação sem infecção e falha em encontrar dois dos outros critérios; foi considerada em qualquer paciente que necessitasse do uso de órtese por longo tempo para proteger uma área deficiente do regenerado ósseo ou do docking site (encontro dos fragmentos ósseos); mau: não consolidação da pseudartrose e persistência ou recorrência da infecção óssea.

\section{3) Complicações}

As complicações foram divididas em: complicações menores: aquelas que foram resolvidas completamente, sem a necessidade de cirurgia adicional antes de terminar o tratamento; complicações maiores sem seqüelas (obstáculos): as que ocorreram durante o período do alongamento e/ou consolidação e foram resolvidas completamente com uma cirurgia adicional antes do término do tratamento; complicações 
maiores: discrepância do comprimento dos membros maior de $2,5 \mathrm{~cm}$, mau alinhamento ósseo maior que $5^{\circ}$, contratura articular maior do que $5^{\circ}$ e persistência da pseudartrose e/ou infecção.

\section{4) Método estatístico}

$\mathrm{Na}$ análise estatística dos resultados foram aplicados os seguintes testes: teste $t$ de Student, análise de variância, teste post-hoc de Duncan, coeficiente de correlação de Pearson. Em todos os testes, fixou-se em 0,05 ou $5 \%($ alfa $=0,05)$ o nível de rejeição da hipótese de nulidade.

\section{RESULTADOS}

Os resultados obtidos foram avaliados e divididos em: resultados funcionais, resultados ósseos, tempo de uso do aparelho e suas correlações, e complicações. O tempo de seguimento dos pacientes variou de 10 a 72 meses, com média de 31,4 meses.

\section{Resultados funcionais}

Dos pacientes, 10 (50\%) negaram qualquer intensidade de dor após terminado o tratamento, cinco pacientes $(25 \%)$ referiram dor leve $(+)$, dois $(10 \%)$ com dor moderada $(++)$ e três $(15 \%)$ referiram dor mais intensa (+++). Apenas dois pacientes $(10 \%)$ necessitaram do uso de órtese após o tratamento. Esses pacientes não apresentavam mobilidade no foco da pseudartrose, mas se sentiam mais confiantes usando a órtese para as atividades diárias. A avaliação da mobilidade articular mostrou que 18 pacientes (90\%) tinham arco de movimento completo no joelho, sete (35\%) tinham alguma restrição na mobilidade do tornozelo e, em apenas um (5\%), as articulações do pé estavam com mobilidade reduzida. Apenas sete (35\%) pacientes referiram alguma restrição nas atividades diárias. Entre os pacientes, 13 (65\%) negaram qualquer limitação das atividades diárias após o tratamento.

Segundo os critérios de Paley ${ }^{(5)}$, o resultado final foi excelente em $50 \%$ dos casos, bom em $40 \%$, regular em $5 \%$ e mau em $5 \%$ dos pacientes.

\section{Resultados ósseos}

Os resultados ósseos foram avaliados separadamente e estão distribuídos conforme: a pseudartrose e suas correlações; o segmento alongado e suas correlações; os desvios angulares; a discrepância no comprimento dos membros inferiores e o resultado final.

Ao final do tratamento, todos os 20 pacientes (100\%) tiveram sucesso e consolidação da pseudartrose. O tempo de consolidação variou de três a 18 meses, com média de 7,6 meses. O tempo de consolidação aumentou proporcionalmente ao grau de gravidade da pseudartrose. Enxertia óssea foi necessária em apenas dois pacientes. $\mathrm{Na}$ amostra analisada, não houve correlação estatisticamente significante entre a idade, o sexo, o lado da lesão, o número de cirurgias prévias, o local da pseudartrose, o tipo e atividade da infecção nos pacientes (tabela 1). No entanto, houve correlação estatisticamente significante entre o tipo de corticotomia e o tempo médio, em meses, de consolidação da pseudartrose. Nas corticotomias em que foi usada serra de Gigli, a consolidação ocorreu em 9,6 meses contra 5,7 meses com o uso de perfurações $(p=0,02)$. Também houve correlação estatisticamente significante entre a extensão da perda óssea e o tempo médio, em meses, de consolidação da pseudartrose $(\mathrm{p}=0,048)$.

Na amostra analisada, não houve correlação estatisticamente significante entre a idade dos pacientes e o tempo médio em meses, de consolidação do segmento alongado; entretanto, houve correlação estatisticamente significante entre o tipo de corticotomia, osteótomo ou serra de Gigli (8,4 contra 8,2 meses, respectivamente) com o tempo médio de consolidação. $\mathrm{O}$ tempo médio de consolidação do segmento alongado também foi proporcional à perda óssea em centímetros.

Nos resultados obtidos, $60 \%$ dos pacientes não tiveram dismetria de membros e/ou desvios angulares e cinco pacientes apresentaram deformidade em valgo de até $5^{\circ}$. Um paciente evoluiu com recurvato de $7^{\circ}$. Apenas um paciente teve encurtamento acima de $3 \mathrm{~cm}$.

Os resultados ósseos finais do tratamento da pseudartrose unilateral infectada da tíbia foram excelentes em oito pacientes (40\%), bons em 10 pacientes (50\%), regulares em um (5\%) e maus em um $(5 \%)$.

\section{Tempo de uso do fixador externo e suas correlações}

O tempo do uso do aparelho de Ilizarov variou de seis a 18 meses, com média de 10,7 meses. Nenhuma diferença estatisticamente significativa foi encontrada entre o tempo de uso do aparelho e as seguintes variáveis: tipo de pseudartrose, idade, sexo, cor, lado, tipo de exposição, número de cirurgias prévias, local da pseudartrose, tipo de infecção e tipo de corticotomia. No entanto, houve correlação estatisticamente significante entre a extensão da perda óssea total em centímetros e o tempo médio, em meses, de uso do fixador externo ( $\mathrm{p}=$ 0,003). Nas perdas ósseas, após o desbridamento, acima de $5 \mathrm{~cm}$, o tempo do uso do aparelho foi maior. 


\section{Complicações}

As complicações estão distribuídas nas tabelas e dividemse em complicações maiores e menores (tabela 2).

TABELA 2

Complicações

\begin{tabular}{|c|c|c|c|}
\hline $\begin{array}{l}\text { Complicações } \\
\text { maiores }\end{array}$ & $\begin{array}{l}\text { Freqüência } \\
\text { absoluta }\end{array}$ & $\begin{array}{l}\text { Complicações } \\
\text { menores }\end{array}$ & $\begin{array}{l}\text { Freqüência } \\
\text { absoluta }\end{array}$ \\
\hline $\begin{array}{l}\text { Discrepância } \\
\text { comprimento } \\
>2,5 \mathrm{~cm}\end{array}$ & 1 & $\begin{array}{l}\text { Infecção } \\
\text { superficial }\end{array}$ & 8 \\
\hline $\begin{array}{l}\text { Mau alinhamento } \\
>5^{\circ}\end{array}$ & 8 & $\begin{array}{l}\text { Contratura do } \\
\text { tornozelo }\end{array}$ & 6 \\
\hline $\begin{array}{l}\text { Contratura do } \\
\text { joelho }>5^{\circ}\end{array}$ & 1 & Edema & 6 \\
\hline $\begin{array}{l}\text { Contratura } \\
\text { tornozelo }>5^{\circ}\end{array}$ & 6 & $\begin{array}{l}\text { Mau alinhamento } \\
\text { do regenerado }\end{array}$ & 2 \\
\hline Infecção óssea & 1 & $\begin{array}{l}\text { Atraso na } \\
\text { consolidação do } \\
\text { regenerado }\end{array}$ & 4 \\
\hline
\end{tabular}

\section{DISCUSSÃO}

O método da distração osteogênica difundido por Ilizarov(2), nos anos 80, usando um fixador externo circular, proporciona liberdade funcional ao paciente, corrige as deformidades e o encurtamento e cura a infecção, por meio da ressecção do osso desvitalizado seguida do transporte gradual de um fragmento ósseo após corticotomia ${ }^{(5,11-12,18)}$. Autores afirmam que, para erradicar a infecção, há necessidade da ressecção dos tecidos desvitalizados e infectados ${ }^{(5-6,10,13,18)}$. Acreditamos que a maior desvantagem da técnica do transporte são o longo tempo de fixação, as complicações no sítio da distração (regenerado ósseo) e no local do encontro dos fragmentos ósseos ${ }^{(5)}$.

Fundamentados na experiência de Ilizarov et $a l^{(2)}$, Gibel et $a l^{(14)}$ e Saleh et $a l^{(15)}$, temos utilizado a técnica de ressecção dos tecidos desvitalizados com posterior encurtamento agudo no foco e alongamento ósseo para o tratamento da pseudartrose infectada da tíbia com defeito ósseo entre 3 e $7 \mathrm{~cm}$. Acreditamos que a maior desvantagem do encurtamento agudo é o risco neurovascular e não recomendamos essa técnica em pacientes com deficiência circulatória. Outra desvantagem é que o encurtamento agudo requer mais ressecção do tecido cicatricial, pois a fibrose tecidual limita a quantidade de encurtamento e o contato entre os extremos ósseos. A ressecção adicional da fíbula aumenta a magnitude do procedimento e desestabiliza completamente o membro.
Nossa hipótese baseia-se na constatação de que os extremos ósseos limpos, cruentos e viáveis, permitem ótima congruência e contato, mantendo o alinhamento adequado e evitando a interposição dos tecidos moles.

Como descrevemos anteriormente, a falha óssea, após as ressecções extensivas, torna-se maior a cada insucesso cirúrgico, tornando-se um problema complexo na sua resolução. A maioria dos autores que utilizam o método de Ilizarov acredita que é difícil o tratamento da pseudartrose infectada da tíbia com perda óssea maior que $3 \mathrm{~cm}^{(5-6,12-14,16)}$.

Devemos lembrar que, em nosso estudo, a média da extensão total da perda óssea foi de $4,65 \mathrm{~cm}$ e que o revestimento cutâneo sobre a área da pseudartrose era de má qualidade, sem exposição óssea em $45 \%$ dos casos e com exposição óssea e sem cobertura cutânea em toda a extensão da área lesada em $25 \%$. Dendrinos ${ }^{(13)}$ e Green ${ }^{(15)}$ encontraram perda óssea total em média de aproximadamente $5 \mathrm{~cm}$.

$\mathrm{O}$ intervalo médio para iniciar o alongamento ósseo nas corticotomias realizadas por perfurações e osteótomo foi de 8,7 e 9,6 dias para aquelas realizadas com serra de Gigli. Acreditamos que o período de latência, antes de iniciar o alongamento, seja muito importante, pois permite a recanalização dos vasos sanguíneos lesados ${ }^{(17)}$. A velocidade do alongamento ósseo foi de $1 \mathrm{~mm}$ ao dia, modificável na presença de regenerados pobres, quando então era diminuída para $0,5 \mathrm{~mm}$ ao dia.

Em nosso estudo, o tempo mínimo de uso do aparelho foi de seis meses e o máximo, de 18 meses, com a média de 10,75 meses, o que está de acordo com os dados da literatura atual ${ }^{(11-}$ ${ }^{12)}$. O momento indicado para retirada do fixador externo permanece sendo de difícil determinação. Não existem, até o momento, técnicas por imagens que permitam a certeza da consolidação óssea e da resistência do calo ósseo ou do regenerado.

A consolidação da pseudartrose ocorreu em todos os pacientes por nós estudados, o que está de acordo com a maioria dos autores que relatam alta frequiência na consolidação quando utilizam o método de Ilizarov. O tempo mínimo para a consolidação dos nossos pacientes foi de três meses e o máximo, de 18 meses, com média de 7,6 meses, inferior ao tempo médio de 10,6 meses dos pacientes tratados por transporte ósseo no trabalho de Paley et $a l^{(5)}$.

No tocante à infecção na pseudartrose, após o tratamento, constatou-se presença em somente 5\% dos casos. A resolução da infecção nos $95 \%$ restantes foi um dos aspectos positivos de nossa casuística e semelhante à encontrada na literatura( ${ }^{(17-18)}$.

Observamos que $60 \%$ dos nossos pacientes apresentavam os membros equalizados ao final do tratamento e que não 
houve encurtamento superior a $2 \mathrm{~cm}$. Outros autores encontraram dismetrias inferiores a $3 \mathrm{~cm}$ na grande maioria dos pacientes estudados $^{(6,17)}$.

\section{CONCLUSÕES}

No presente estudo, a metodologia de Ilizarov, associada ao encurtamento agudo no foco da fratura, com subsequiente alongamento, mostrou-se eficaz para o tratamento da pseudartrose infectada da tíbia. Nas corticotomias, em que foi usada serra de Gigli, a consolidação sofreu retarde quando comparada com a osteotomia por múltiplas perfurações por broca. O tempo de consolidação do foco de pseudartrose e o tempo de formação do segmento ósseo alongado foram proporcionais à extensão da perda óssea.

\section{REFERÊNCIAS}

1. Campbell WC, Crenshaw AH. Campbell's operative orthopaedics. 7a ed. St. Louis: Mosby; 1987.

2. Ilizarov GA, Devyatov AA, Kamerin VK. Plastic reconstruction of longitudinal bone defects by means of compression and subsequent distraction. Acta Chir Plast. 1980;22(1):32-41.

3. Cierny G 3rd, Zorn KE. Segmental tibial defects. Comparing conventional and Ilizarov methodologies. Clin Orthop Relat Res. 1994; (301):118-23.

4. Marsh JL, Prokuski L, Biermann JS. Chronic infected tibial nonunions with bone loss. Conventional techniques versus bone transport. Clin Orthop Relat Res. 1994;(301):139-46.

5. Paley D, Catagni MA, Argnani F, Villa A, Benedetti GB, Cattaneo R. Ilizarov treatment of tibial nonunions with bone loss. Clin Orthop Relat Res. 1989;(241):146-65.
6. Cattaneo R, Catagni M, Johnson EE. The treatment of infected nonunions and segmental defects of the tibia by the methods of Ilizarov. Clin Orthop Relat Res. 1992;(280):143-52.

7. Catagni MA, Felici JVN. Alongamento de dois níveis e o método de Ilizarov (trifocal) no tratamento da pseudoartrose tibial com perda óssea. Rev Bras Ortop. 1996;31(8):613-9.

8. Catagni MA. Classificazione e trattamento delle pseudartrosi di gamba senza perdita di sostanza ossea. Atti del 1ํㅡㄹ Congresso ASAMI; 1986. p. 95-7.

9. Muller ME, Allgower M, Schneider R, Willenegger H, editors. Manual of internal fixation: techniques recommended by the AO-ASIF group. 3rd ed. Berlin: Springer-Verlag; 1991.

10. Fischgrund J, Paley D, Suter C. Variables affecting time to bone healing during limb lengthening. Clin Orthop Relat Res. 1994;(301):31-7.

11. Green SA, Jackson JM, Wall DM, Marinow H, Ishkanian J. Management of segmental defects by the Ilizarov intercalary bone transport method. Clin Orthop Relat Res. 1992;(280):136-42.

12. Dagher F, Roukoz S. Compound tibial fractures with bone loss treated by the Ilizarov technique. J Bone Joint Surg Br. 1991;73(2):316-21.

13. Tuffi GJ, Bongiovanni JC, Mestriner LA. Tratamento das pseudartroses infectadas da tíbia com falhas ósseas pelo método de Ilizarov, utilizando o transporte ósseo. Rev Bras Ortop. 2001;36(8):292-300.

14. Dendrinos GK, Kontos S, Lyritsis E. Use of the Ilizarov technique for treatment of non-union of the tibia associated with infection. J Bone Joint Surg Am. 1995;77(6):835-46.

15. Catena RS, Targa WHC, Bongiovanni JC, Nery CAS, Laredo Filho J, Catena ACS. Tratamento da pseudoartrose traumática infectada da diáfise da tíbia pelo método de Ilizarov. Rev Bras Ortop. 1998;33(8):583-7.

16. Green SA. Ilizarov method. Clin Orthop Relat Res. 1992;(280):2-6.

17. Aronson J, Johnson E, Harp JK. Local bone transportation for treatment of intercalary defects by the Ilizarov technique. Biomechanical and clinical considerations. Clin Orthop Relat Res. 1989;(243):71-9.

18. Delloye C, Delefortrie G, Coutelier L, Vincent A. Bone regenerate formation in cortical bone during distraction lengthening. An experimental study. Clin Orthop Relat Res. 1990;(250):34-42. 